\title{
Optimisation de la biométhanisation des effluents de manioc issus de la filière de fabrication de l'attiéké (semoule de manioc)
}

\author{
Nazo Edith KPATA-KONAN ${ }^{1,2}$, Koffi Félix KONAN ${ }^{2,3,4 *}$, Martin KOUAME \\ KOUAME $^{2,3}$, Yao Francis KOUAME ${ }^{1,2}$, Théophile GNAGNE ${ }^{2,5}$ et Kablan TANO ${ }^{6}$ \\ ${ }^{I}$ Laboratoire des Sciences de l'Environnement, UFR des Sciences et Gestion de l'Environnement, Université \\ Abobo-Adjamé, 02 BP 801 Abidjan 02, Côte d'Ivoire. \\ ${ }^{2}$ Centre Régional pour l'Eau Potable et l'Assainissement à Faible coût, Représentation Nationale de Côte \\ d'Ivoire, 18 BP 80 Abidjan 18, Côte d'Ivoire. \\ ${ }^{3}$ Laboratoire d'Environnement et de Biologie Aquatique, UFR des Sciences et Gestion de l'Environnement, \\ Université Abobo-Adjamé, 02 BP 801 Abidjan 02, Côte d'Ivoire. \\ ${ }^{4}$ Unité Pédagogique et de Recherche de Biologie et Physiologie Animales, Unité Régionale d'Enseignement \\ Supérieur de Daloa, BP 150 Daloa, Côte d'Ivoire. \\ ${ }^{5}$ Laboratoire Géoscience et Environnement, UFR des Sciences et Gestion de l'Environnement, Université \\ Abobo-Adjamé, 02 BP 801 Abidjan 02, Côte d'Ivoire. \\ ${ }^{6}$ Laboratoire de Technologie Alimentaire des Produits Tropicaux, UFR des Sciences et Techniques \\ Alimentaires, Université Abobo-Adjamé, 02 BP 801 Abidjan 02, Côte d'Ivoire. \\ *Auteur correspondant ; E-mail : konanfelix@yahoo.fr; Tél. : 0022506 216160.
}

\section{RESUME}

Cette étude s'est penchée sur les conditions optimales de biodigestion des effluents de manioc de la filière attiéké (semoule de manioc). Ces effluents proviennent de la fabrique d'attiéké d'Azito (village Ebrié de la commune de Yopougon dans le district d'Abidjan). Les six digesteurs expérimentaux utilisés, ont été alimentés comme suit : 1) effluent de manioc;2) effluent + bouse de vache ; 3) effluent + urine ; 4-5 et 6) effluent + urine + différentes quantités de bouse de vache. Aux résultats, les digesteurs ont fonctionné de façon mésophile avec des températures comprises entre 24,0 et $35,6{ }^{\circ} \mathrm{C}$. Les digesteurs sans urine ont un $\mathrm{pH}$ variant entre 3 et 4 durant l'expérimentation. Pour ceux contenant de l'urine humaine, le pH a oscillé entre 6,46 et 10,29. Concernant le volume de gaz produit, il est significativement plus important dans les digesteurs contenant de l'urine que dans les digesteurs sans ajout d'urine. Le test d'inflammabilité du gaz produit est positif sauf pour le premier digesteur. Dans l'ensemble, les résultats de l'étude montrent que l'ajustement du $\mathrm{pH}$ avec l'urine humaine avant le démarrage du traitement des effluents de manioc issus de la filière de fabrication de l'attiéké par digestion anaérobie, est nécessaire pour l'optimisation du processus de biométhanisation.

(c) 2011 International Formulae Group. All rights reserved.

Mots clés : Digestion anaérobie, effluents de manioc, biogaz.

\section{INTRODUCTION}

En Côte d'Ivoire, l'attiéké (semoule de manioc) est la principale forme d'utilisation alimentaire des racines tubéreuses de manioc
(Kakou, 2000). Cependant, la transformation de la pulpe de manioc en attiéké est une opération génératrice de déchets solides et

(C) 2011 International Formulae Group. All rights reserved.

DOI : http://dx.doi.org/10.4314/ijbcs.v5i6.13 
liquides. Les sous-produits liquides (effluents) sont riches en matière organique (Colin et al., 2007 ; Ubalua, 2007) et très toxiques du fait de la teneur élevée en cyanure (Asiedu, 1991 ; Ihedioha, 2002). Ces effluents constituent une source importante de pollution dans la ville d'Abidjan. En effet, les femmes des villages Ebrié engloutis par l'expansion de la ville d'Abidjan ont gardé leur traditionnelle activité de production d'attiéké dont les résidus de fabrication, notamment le jus issu du pressage du manioc, sont rejetés dans la nature sans traitement préalable. Pourtant, le contrat d'affermage entre l'Etat Ivoirien et la Société de Distribution d'Eau en Côte d'Ivoire (SODECI), chargée de l'assainissement, ne prend pas en compte les pollutions diffuses et ponctuelles issues des activités d'industries agro-alimentaires artisanales. De ce fait, un procédé naturel de traitement et de détoxication des effluents de manioc devient fondamental. Ainsi, dans le souci de valoriser lesdits effluents et de protéger l'environnement, un processus de biodigestion anaérobie a été mis en place pour la transformation de la matière organique en énergie par les bactéries méthanogènes en l'absence d'oxygène (Kalloum et al., 2007). Ces effluents sont stockés dans des enceintes confinées (digesteurs) à l'intérieur desquels les réactions de fermentation sont optimisées et contrôlées. Ce processus biologique permet de produire du biogaz combustible, une énergie renouvelable, à partir de matière organique par des bactéries dans des conditions anaérobies (Le Hyaric et al., 2010; Kalloum et al., 2011; Zhu et al., 2011). Selon Gomez-Lahoz et al. (2007) et Poh et Chong (2009), cette digestion anaérobie peut réduire de moitié la charge polluante organique. Le digesta est selon Gomez-Lahoz et al. (2007) et Kalloum et al. (2011), stable, indolore, débarrassé en majeure partie des germes pathogènes et peut même être utilisé comme amendements des sols pour l'agriculture. Dans le cas du traitement des effluents biorécalcitrants et pour booster la production de biogaz, la co-digestion apparait comme une alternative efficace pour optimiser et contrôler la fermentation anaérobie (Mata-Alvarez et al., 2000 ; Kpata, 2005 ; Davidsson et al., 2008 ; Luostarinen et al., 2009 ; Fountoulakis et al., 2010 ; Montusiewicz et Lebiocka, 2011 ; Zhu et al., 2011). En effet, selon ces auteurs, la co-digestion anaérobie a l'avantage d'améliorer le ratio de nutriments dans les substrats mixtes et d'ajuster le $\mathrm{pH}$ du milieu réactionnel pour un traitement plus efficace de la pollution organique et la production de biogaz.

Plusieurs paramètres tels que le $\mathrm{pH}$, la température et la composition du substrat régissent le bon fonctionnement d'un digesteur anaérobie (Parawira et al., 2006; Colin et al., 2007 ; Bouallagui et al., 2009 ; Fountoulakis et al., 2010). La baisse du pH jusqu'en dessous de 4 implique inévitablement une inhibition significative de la phase méthanogénèse (Mahan, 2004 ; Amir, 2005 ; Kpata, 2005). Cette dernière est l'étape limitant le processus de digestion anaérobie qui comporte généralement quatre phases successives (Héteu et Martin, 2003 ; Cresson, 2006): l'hydrolyse et l'acidogenèse qui se traduisent par la formation des acides gras volatils, l'acétogénèse, responsable de la formation d'acide acétique, d'hydrogène et de gaz carbonique, et enfin la méthanogénèse qui transforme l'acétate en méthane $\left(\mathrm{CH}_{4}\right)$. Cette dernière phase exige des conditions bien précises pour son déclenchement.

Le présent travail vise à déterminer les conditions optimales de biodigestion des effluents de manioc de la filière attiéké par voie anaérobie à travers le suivi du $\mathrm{pH}$, de la température et de la production de biogaz.

\section{MATERIEL ET METHODES \\ Matériel}

Le dispositif expérimental est composé de six digesteurs constitués chacun de deux fûts métalliques de capacité $100 \mathrm{~L}$ et $186 \mathrm{~L}$ 
ouverts chacun sur un des cotés (Figure 1). Le grand fût contient le milieu réactionnel. Le plus petit fût est plongé dans le plus grand jusqu'à toucher le fond de ce dernier. Le plus petit fût sert de gazomètre où est stocké le biogaz produit. Sur ce fût, sont montés un malaxeur et une vanne. La vanne permet d'éviter la sortie du biogaz produit au fur et à mesure que la fermentation se déroule. Quant au malaxeur, il sert à homogénéiser le milieu réactionnel de sorte à éviter la décantation.

La matière première utilisée dans cette étude est l'effluent issu de la fabrique d'attiéké d'Azito, village Ebrié de la commune de Yopougon (District d'Abidjan). Cet effluent dont le $\mathrm{pH}$ varie entre 3,00 et 4,20 est constitué du jus de pressage et de l'eau de lavage du manioc. La bouse de vache et l'urine humaine dont le $\mathrm{pH}$ est compris entre 8,00 et 10,00 ont été respectivement utilisées pour ensemencement et neutralisation du milieu réacteur. Cette urine provient des latrines sèches à déviation d'urine et des urinoirs conçus par la Représentation Nationale de Côte d'Ivoire du Centre Régional pour l'Eau Potable et l'Assainissement à faible coût (CREPA_RN Côte d'Ivoire). La bouse de vache provient de l'abattoir municipal de Yopougon sis à Azito.

La température et le $\mathrm{pH}$ du milieu réactionnel ont été mesurés à l'aide d'un pH-mètre de type WTW (pH 3210 SET 2). Une balance commerciale de type CAP.20 kg/GRAD.50 g a été utilisée pour la pesée de la bouse de vache.

\section{Méthodes}

Les essais ont eu lieu entre juillet et octobre 2010. Les six digesteurs mis en place ont été alimentés comme suit :

- digesteur 1: 124 L d'effluent de manioc ;

- digesteur 2: $124 \mathrm{~L}$ d'effluent de manioc $+10 \mathrm{~kg}$ de bouse de vache ;
- digesteur 3: $70 \mathrm{~L}$ d'effluent de manioc +54 L d'urine ;

- digesteur 4: $70 \mathrm{~L}$ d'effluent de manioc $+54 \mathrm{~L}$ d'urine $+5 \mathrm{~kg}$ de bouse de vache ;

- digesteur 5: $70 \mathrm{~L}$ d'effluent de manioc $+54 \mathrm{~L}$ d'urine $+10 \mathrm{~kg}$ de bouse de vache ;

- digesteur 6: 70 L d'effluent de manioc $+54 \mathrm{~L}$ d'urine $+15 \mathrm{~kg}$ de bouse de vache.

Pour les digesteurs 3, 4, 5 et 6, les milieux réactionnels ont été tamponnés à $\mathrm{pH} 7$ à l'aide de l'urine humaine. L'ensemble des digesteurs, exposés au soleil, fonctionne de façons discontinues.

Le volume $(\mathrm{V})$ du biogaz produit a été déterminé à partir de l'expression : $\mathrm{V}=\pi \mathrm{R}^{2} \mathrm{H}$, après mesure de la hauteur $(\mathrm{H}) \mathrm{de}$ soulèvement du gazomètre (petit fût); $\mathrm{R}=$ Rayon du gazomètre (petit fût).

L'échantillonnage de l'effluent est réalisé à l'entrée et à la sortie des différents digesteurs. Le $\mathrm{pH}$, la température et le volume de gaz produit sont mesurés in situ de façon journalière.

Pour l'analyse statistique, la nature des données a été premièrement déterminée afin de savoir laquelle des méthodes paramétriques ou non paramétriques est convenable. Le test de Shapiro-Wilk (W) a été utilisé pour tester la normalité de la distribution au seuil de 0.05. L'ANOVA a été conduite pour faire ressortir les éventuelles différences entre digesteurs en fonction des variables considérées. Les analyses statistiques ont été réalisées par le logiciel Paleotological Statistic (PAST) version 2.10 (Hammer et al., 2001).

\section{RESULTATS}

\section{Température}

Les températures enregistrées varient entre 25,5 et $29,1{ }^{\circ} \mathrm{C}$ pour le réacteur 1 (effluent de manioc) (Figure 2a), entre 24,2 et 
31,3 ${ }^{\circ} \mathrm{C}$ pour le réacteur 2 (effluents de manioc $+10 \mathrm{~kg}$ de bouse de vache) (Figure $2 b)$, entre 25 et $32,2{ }^{\circ} \mathrm{C}$ pour le réacteur 3 (effluent de manioc + urine) (Figure 2c), entre 24,1 et $35,6^{\circ} \mathrm{C}$ pour le réacteur 4 (effluent de manioc + urine $+5 \mathrm{~kg}$ de bouse de vache) (Figure 2d), 24,1 et $35,4{ }^{\circ} \mathrm{C}$ pour le réacteur 5 (effluents de manioc + urine $+10 \mathrm{~kg}$ de bouse de vache) (Figure 2e), et entre 24 et $35,1^{\circ} \mathrm{C}$ pour le réacteur 6 (effluents de manioc + urine $+15 \mathrm{~kg}$ de bouse de vache) (Figure $2 \mathrm{f}$ ).

Dans l'ensemble, bien que les digesteurs aient fonctionné de façon mésophile, la température dans les digesteurs contenant de l'urine $(3,4,5$ et 6$)$ est significativement $(\mathrm{p}<0.05)$ plus élevée que celle dans les digesteurs sans urine (1 et 2) (Tableau 1).

\section{pH}

Les digesteurs sans urine (Figure $3 \mathrm{a}$ et $3 b)$ ont un $\mathrm{pH}$ qui varie entre 3 et 4 durant l'expérimentation. Dans le réacteur 1 (effluent de manioc) le $\mathrm{pH}$ augmente légèrement de 3,71 à 4,01 puis se stabilise autour de 4,5 à partir du $7^{\text {ème }}$ jour (Figure $3 a$ ). Au niveau du réacteur 2 (effluents de manioc $+10 \mathrm{~kg}$ de bouse de vache), du $1^{\text {er }}$ jour au $11^{\text {ème }}$ jour, le $\mathrm{pH}$ chute de 4,68 à 3,73 puis augmente progressivement jusqu'à 4,57 à la fin de l'expérimentation (Figure 3b). Dans les digesteurs 3 (effluent de manioc + urine), 4, 5 et 6 (effluents de manioc + urine + bouse de vache), neutralisés à l'urine, le $\mathrm{pH}$ évolue de façon similaire. Les valeurs chutent légèrement de 7 à 6,75 pour le réacteur 3 , de 7 à 6,72 pour le réacteur 4 , de 7,01 à 6,61 pour le réacteur 5 et de 7,03 à 6,46 pour le réacteur 6 , puis augmentent progressivement pour atteindre $8,47,10,29,8,16$ et 9,75 pour les digesteurs 3, 4, 5 et 6 respectivement (Figures 3c, 3d, 3e et 3f).

Globalement, le $\mathrm{pH}$ dans les digesteurs 1 et 2 sans urine est resté significativement ( $p$
$<0.05)$ moins élevé que dans les digesteurs contenant de l'urine (3, 4, 5 et 6$)$ (Tableau 1). Comparativement aux réacteurs sans urine qui ont un milieu acide, ceux neutralisés à l'urine humaine (réacteurs 3, 4, 5 et 6) ont un milieu alcalin tout le long de l'expérimentation.

\section{Volume du biogaz produit}

La production de biogaz est quasiment nulle sur toute la période de fonctionnement du réacteur 1 (effluent de manioc) (Figure 4a). Concernant le digesteur 2 (effluents de manioc $+10 \mathrm{~kg}$ bouse de vache), la production de gaz est intervenue dès le $2^{\text {ème }}$ jour de fonctionnement avec un volume de 6,5 $\mathrm{dm}^{3}$ (Figure $4 \mathrm{~b}$ ). Le volume de gaz produit a atteint un pic de $43,9 \mathrm{dm}^{3}$ le $4{ }^{\text {ème }}$ jour, ensuite il a baissé progressivement jusqu'à s'annuler à partir du $68^{\text {ème }}$ jour. Le test d'inflammabilité du gaz produit par le digesteur 2 est positif après 50 jours de fonctionnement.

Le réacteur 3 (effluent de manioc + urine) a produit $1,6 \mathrm{dm}^{3}$ de gaz dès le $3^{\text {ème }}$ jour de fonctionnement (Figure 4c). La production de gaz dans ce réacteur a connu deux phases : une phase ascendante du $3^{\text {ème }}$ jour de sa mise en place jusqu'au $7^{\text {ème }}$ jour, avec un pic à 60 $\mathrm{dm}^{3}$ et une phase descendante jusqu'au $34^{\text {ème }}$ jour où le volume de gaz chute à zéro. Le test d'inflammabilité du gaz produit par ce réacteur a été positif du $7^{\text {ème }}$ au $34^{\text {ème }}$ jour.

Pour le digesteur 4 (effluent de manioc + urine $+5 \mathrm{~kg}$ de bouse de vache), la production de gaz est observée du $2^{\text {ème jour }}$ jusqu'au $42^{\text {ème }}$ jour de fonctionnement (Figure $4 d)$. Le volume maximal $\left(21,12 \mathrm{dm}^{3}\right)$ de gaz dans ce digesteur a été observé le $14^{\text {ème }}$ jour. Le test d'inflammabilité du gaz produit par le digesteur 4 a été positif à partir du $15^{\text {ème }}$ jour de fonctionnement.

Pour ce qui concerne le réacteur 5 (effluents de manioc + urine $+10 \mathrm{~kg}$ bouse de vache), la production de gaz $\left(61 \mathrm{dm}^{3}\right)$ a été enregistrée dès le $2^{\text {ème }}$ jour de son lancement 
(Figure 4e). Cette production de gaz présente deux pics. Le premier, d'une valeur de 81,8 $\mathrm{dm}^{3}$, intervient le $5^{\text {ème }}$ jour. Le volume de gaz décroit ensuite progressivement jusqu'à 8,7 $\mathrm{dm}^{3}$ le $37^{\text {ème }}$ jour avant d'atteindre le second pic qui apparaît le $75^{\text {ème }}$ jour avec un volume de $64,4 \mathrm{dm}^{3}$. Le test d'inflammabilité du gaz produit par le digesteur 5 est positif à partir du $4^{\text {ème }}$ jusqu'à ce jour.

Quant au digesteur 6 (effluents de manioc + urine $+15 \mathrm{~kg}$ de bouse de vache), la production de gaz $\left(13,93 \mathrm{dm}^{3}\right)$ a été enregistrée dès le $2^{\text {ème }}$ jour de son lancement (Figure 4f). Pour ce réacteur, la production présente trois pics. Le premier de $22,63 \mathrm{dm}^{3}$ est intervenu le $4^{\text {ème }}$ jour. Ce volume décroit ensuite jusqu'à $1,74 \mathrm{dm}^{3}$ le $16^{\text {ème }}$ jour avant de remonter progressivement pour atteindre le second pic qui apparaît le $22^{\text {ème }}$ jour avec un volume de $52,24 \mathrm{dm}^{3}$ de gaz. Le troisième pic d'une valeur de $50,50 \mathrm{dm}^{3}$ apparaît le $39^{\text {ème }}$ jour. Ce volume diminue progressivement jusqu'à s'annuler le $56^{\text {ème }}$ jour. Le test d'inflammabilité du gaz produit par le digesteur 6 a été positif du $19^{\text {ème }}$ jusqu'au $55^{\text {ème }}$ jour de l'expérimentation.

Dans l'ensemble, la production de gaz est significativement $(\mathrm{p}<0.05)$ plus importante dans les digesteurs $(3,4,5$ et 6$)$ contenant de l'urine que celle dans les digesteurs 1 et 2 sans urine (Tableau 1).

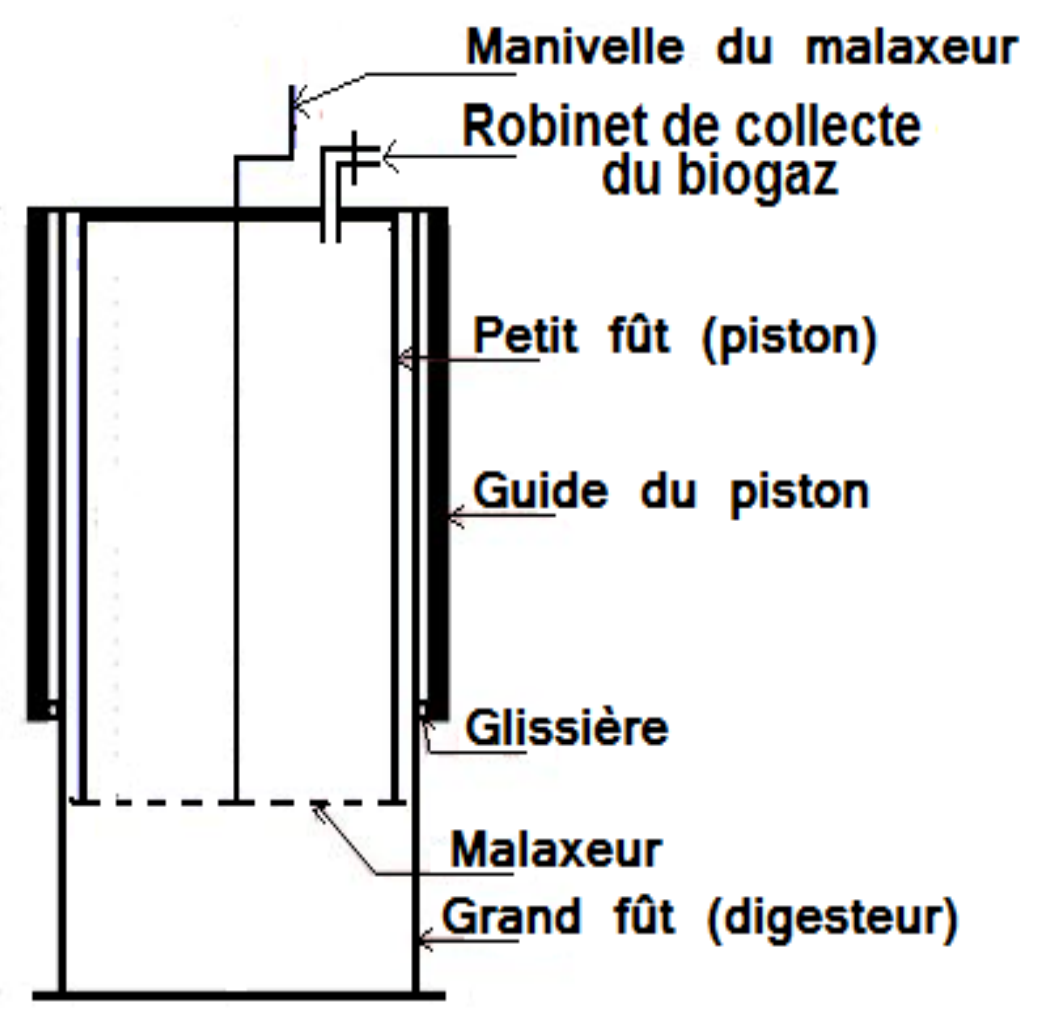

Figure 1 : Schéma présentant une vue de profil du digesteur expérimental. 

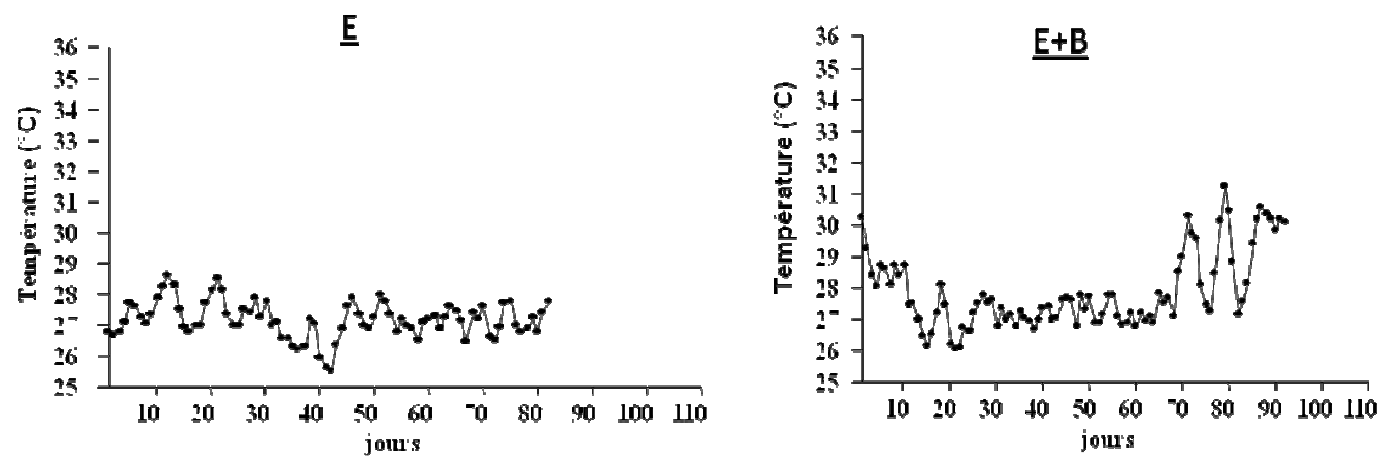

a

b

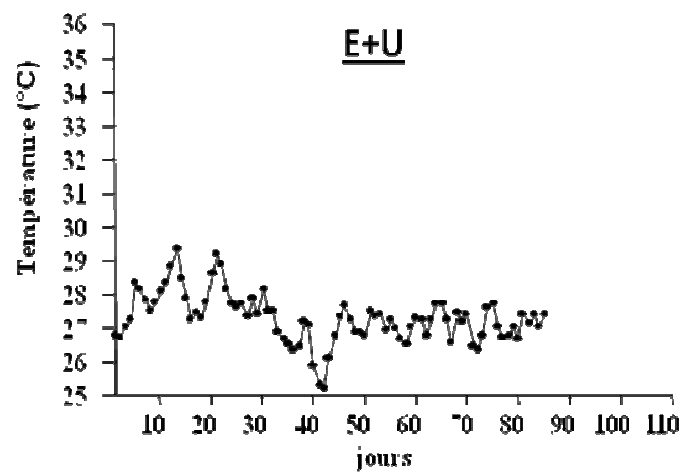

$\underline{E+U+B 5}$

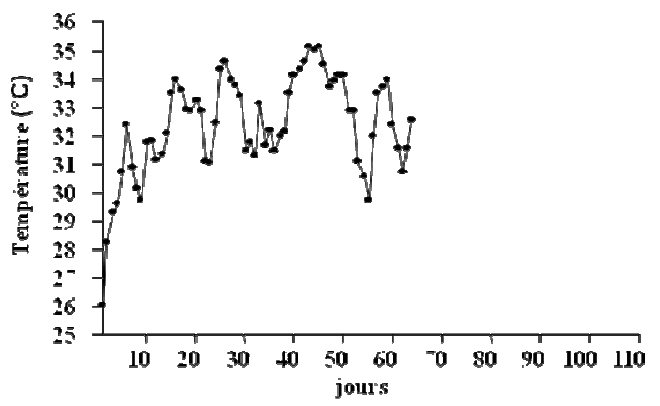

c

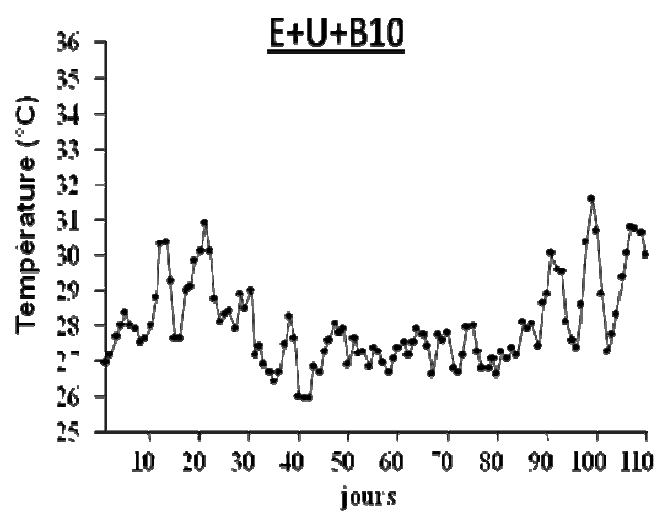

d

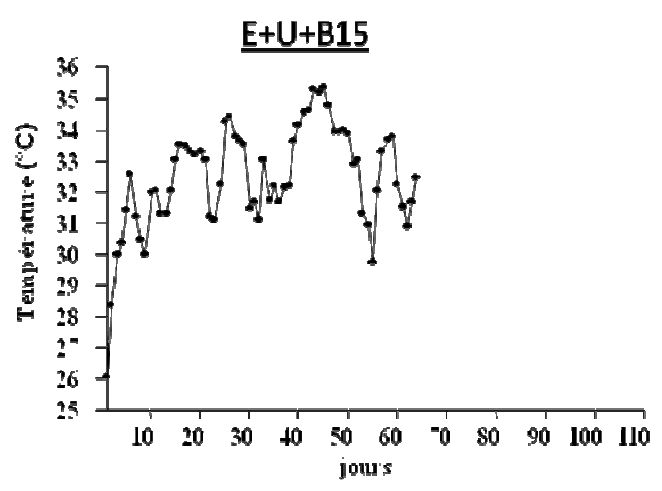

f

Figure 2 : Variation journalière de la température dans les différents réacteurs : a) réacteur $1, \mathrm{~b}$ ) réacteur $2, \mathrm{c}$ ) réacteur $3, \mathrm{~d}$ ) réacteur 4 , e) réacteur 5 , f) réacteur $6 ; \mathrm{E}=$ effluent de manioc, $\mathrm{E}+\mathrm{B}=$ effluents de manioc $+10 \mathrm{~kg}$ de bouse de vache, $\mathrm{E}+\mathrm{U}=$ effluent de manioc + urine, $\mathrm{E}+\mathrm{U}+\mathrm{B} 5=$ effluents de manioc + urine $+5 \mathrm{~kg}$ de bouse de vache, $\mathrm{E}+\mathrm{U}+\mathrm{B} 10=$ effluents de manioc + urine $+10 \mathrm{~kg}$ de bouse de vache, $\mathrm{E}+\mathrm{U}+\mathrm{B} 15=$ effluents de manioc + urine $+15 \mathrm{~kg}$ de bouse de vache. 


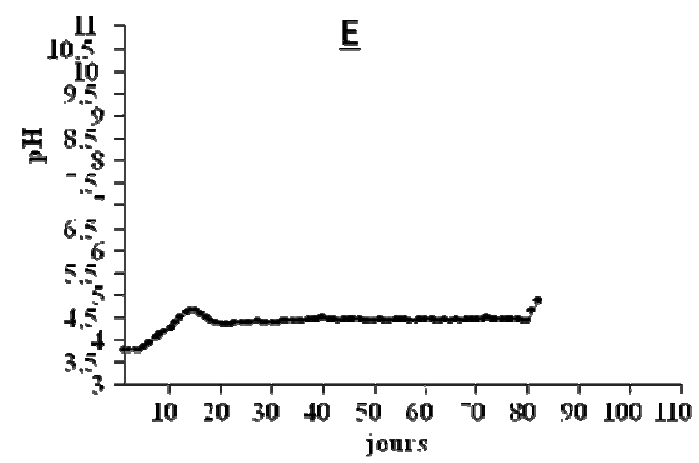

a

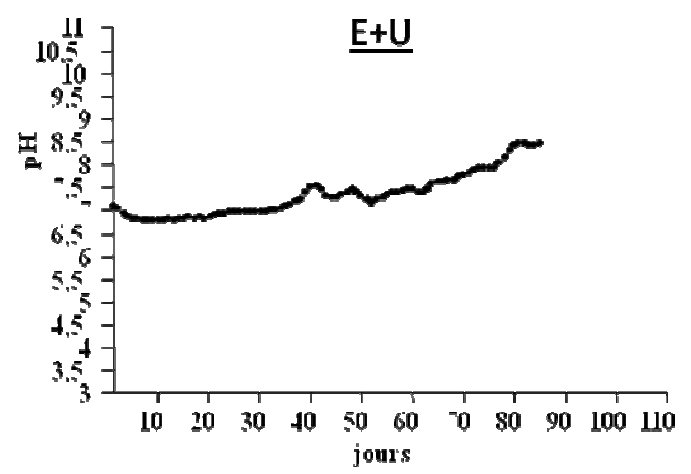

c

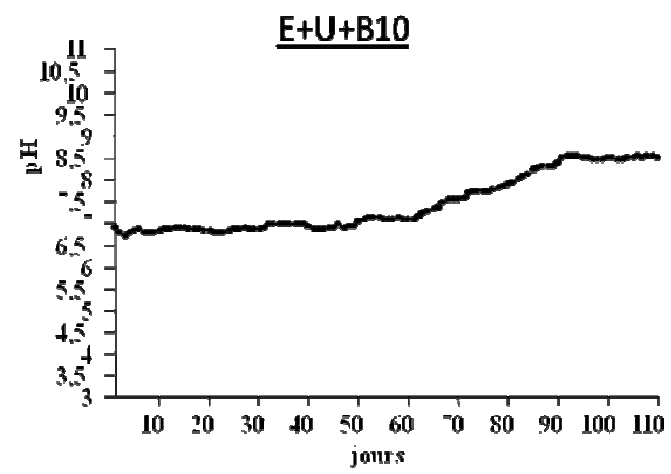

e

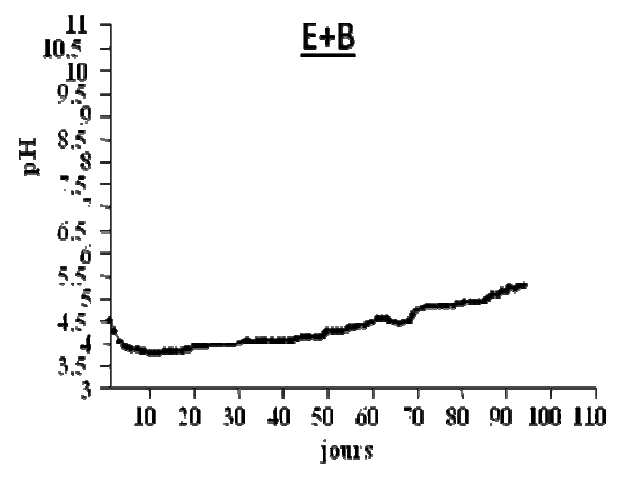

b

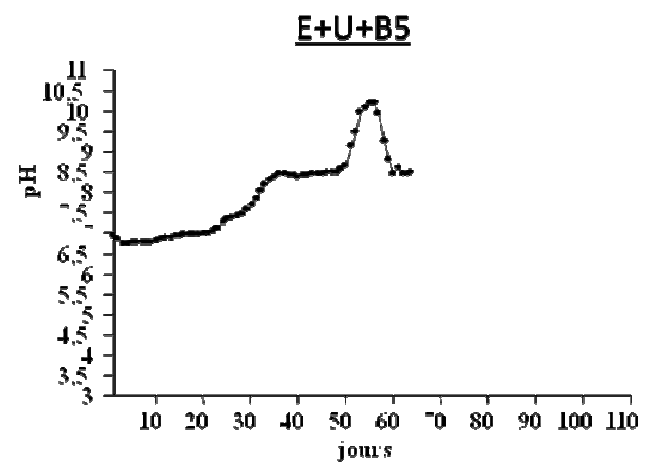

d

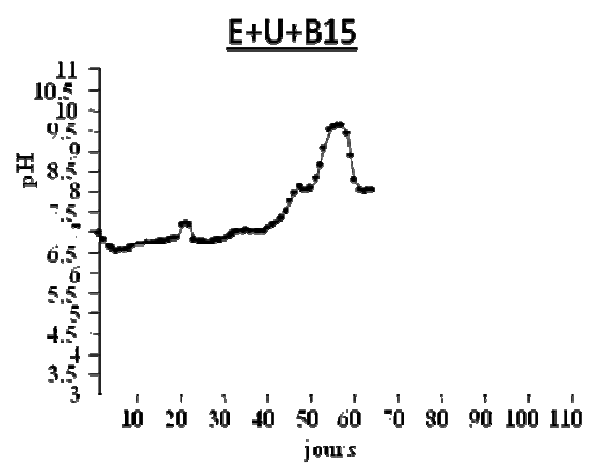

$\mathbf{f}$

Figure 3 : Variation journalière du $\mathrm{pH}$ dans les différents réacteurs : a) réacteur $1, \mathrm{~b}$ ) réacteur 2, c) réacteur $3, \mathrm{~d}$ ) réacteur $4, \mathrm{e}$ ) réacteur $5, \mathrm{f}$ ) réacteur $6 ; \mathrm{E}=$ effluent de manioc, $\mathrm{E}+\mathrm{B}=$ effluents de manioc $+10 \mathrm{~kg}$ de bouse de vache, $\mathrm{E}+\mathrm{U}=$ effluent de manioc + urine, $\mathrm{E}+\mathrm{U}+\mathrm{B} 5=$ effluents de manioc + urine $+5 \mathrm{~kg}$ de bouse de vache, $\mathrm{E}+\mathrm{U}+\mathrm{B} 10=$ effluents de manioc + urine $+10 \mathrm{~kg}$ de bouse de vache, $\mathrm{E}+\mathrm{U}+\mathrm{B} 15=$ effluents de manioc + urine $+15 \mathrm{~kg}$ de bouse de vache. 

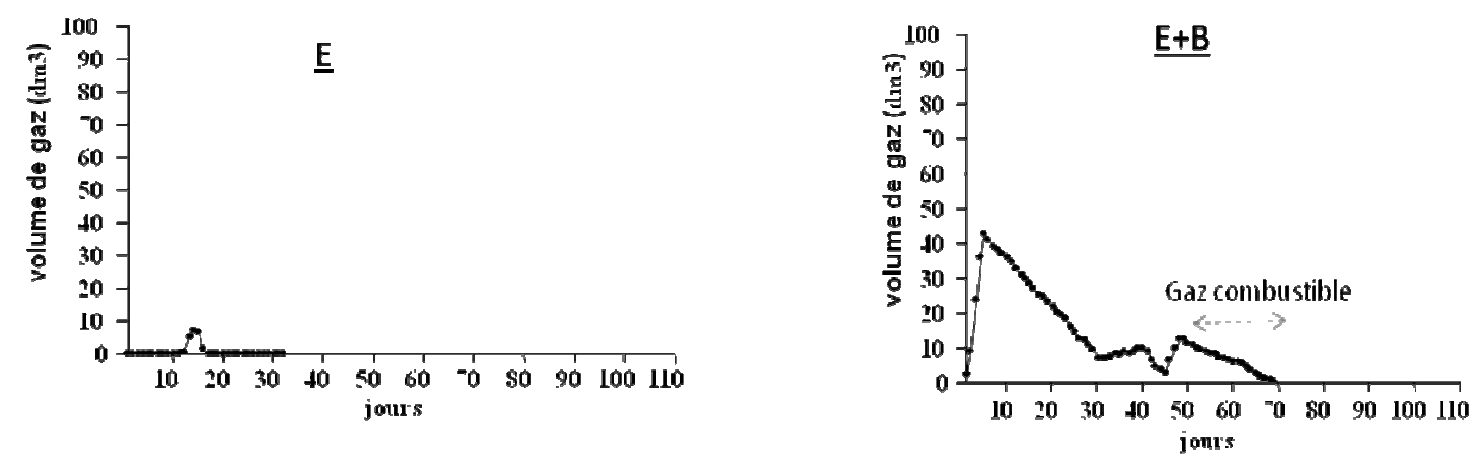

a

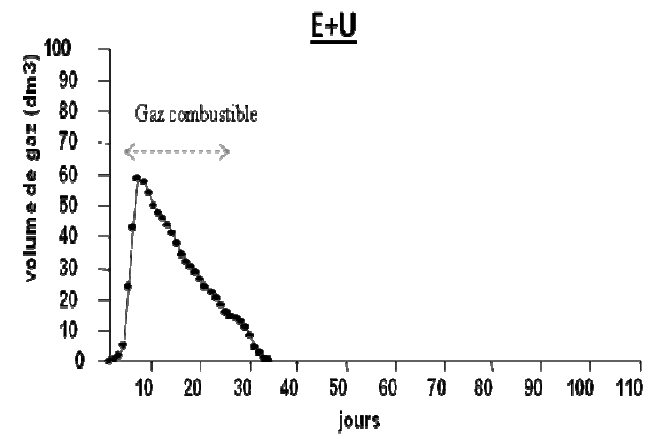

c

\section{$\underline{E+U+B 10}$}

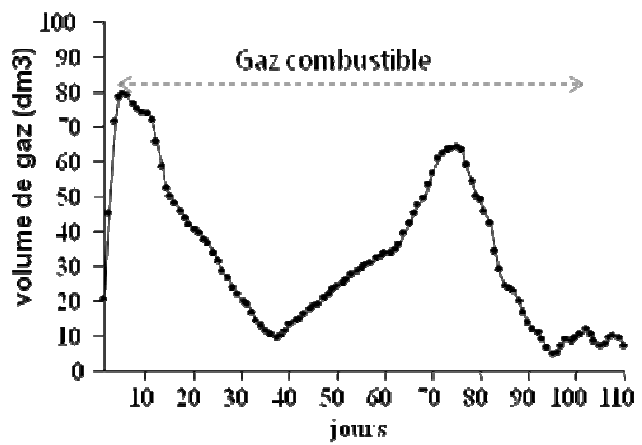

e b

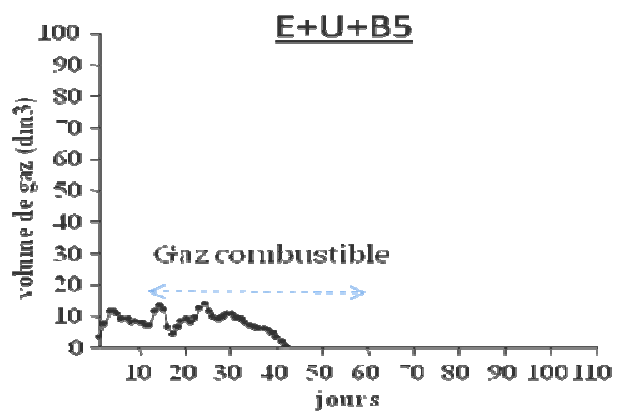

d

\section{$\underline{\mathrm{E}+\mathrm{U}+\mathrm{B} 15}$}

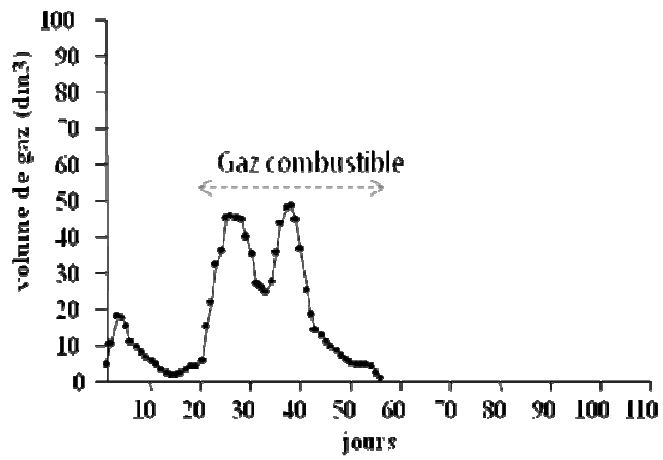

$\mathrm{f}$

Figure 4 : Variation journalière de la production de gaz par les différents réacteurs : a) réacteur 1, b) réacteur $2, \mathrm{c}$ ) réacteur $3, \mathrm{~d}$ ) réacteur 4, e) réacteur $5, \mathrm{f}$ ) réacteur $6 ; \mathrm{E}=$ effluent de manioc, $\mathrm{E}+\mathrm{B}=$ effluents de manioc $+10 \mathrm{~kg}$ de bouse de vache, $\mathrm{E}+\mathrm{U}=$ effluent de manioc + urine, $\mathrm{E}+\mathrm{U}+\mathrm{B} 5=$ effluents de manioc + urine + $5 \mathrm{~kg}$ de bouse de vache, $\mathrm{E}+\mathrm{U}+\mathrm{B} 10=$ effluents de manioc + urine $+10 \mathrm{~kg}$ de bouse de vache, $\mathrm{E}+\mathrm{U}+\mathrm{B} 15=$ effluents de manioc + urine $+15 \mathrm{~kg}$ de bouse de vache. 
Tableau 1 : Résultats du test paramétrique ANOVA réalisé pour comparer le $\mathrm{pH}$, la température et le volume de gaz produit dans les différents digesteurs.

\begin{tabular}{lcccccc}
\hline Température & $\mathbf{E}$ & $\mathbf{E + B}$ & $\mathbf{E + U}$ & $\mathbf{E + U + B 5}$ & $\mathbf{E + U + B 1 0}$ & $\mathbf{E + U + B 1 5}$ \\
\hline $\mathbf{E}$ & - & $>0.05$ & $>0.05$ & $<0.001$ & $<0.001$ & $<0.001$ \\
$\mathbf{E}+\mathbf{B}$ & & - & $>0.05$ & $<0.01$ & $<0.001$ & $<0.001$ \\
$\mathbf{E}+\mathbf{U}$ & & - & $<0.001$ & $>0.05$ & $<0.001$ \\
$\mathbf{E + U + B 5}$ & & & - & $<0.001$ & $>0.05$ \\
E+U+B10 & & & & - & $<0.001$ \\
E+U+B15 & & & & & & - \\
\hline
\end{tabular}

\begin{tabular}{lcccccc}
\hline $\mathbf{p H}$ & $\mathbf{E}$ & $\mathbf{E}+\mathbf{B}$ & $\mathbf{E}+\mathbf{U}$ & $\mathbf{E}+\mathbf{U}+\mathbf{B 5}$ & $\mathbf{E}+\mathbf{U}+\mathbf{B 1 0}$ & $\mathbf{E}+\mathbf{U}+\mathbf{B 1 5}$ \\
\hline $\mathbf{E}$ & - & $>0.05$ & $<0.001$ & $<0.001$ & $<0.001$ & $<0.001$ \\
$\mathbf{E}+\mathbf{B}$ & & - & $<0.001$ & $<0.001$ & $<0.001$ & $<0.001$ \\
$\mathbf{E}+\mathbf{U}$ & & - & $<0.001$ & $>0.05$ & $>0.05$ \\
$\mathbf{E}+\mathbf{U}+\mathbf{B 5}$ & & & - & $<0.001$ & $<0.001$ \\
$\mathbf{E}+\mathbf{U}+\mathbf{B 1 0}$ & & & & & - & $>0.05$ \\
$\mathbf{E}+\mathbf{U}+\mathbf{B 1 5}$ & & & & & & - \\
\hline
\end{tabular}

\begin{tabular}{|c|c|c|c|c|c|c|}
\hline $\begin{array}{l}\text { Volume de } \\
\text { biogaz }\end{array}$ & $\mathbf{E}$ & $\mathbf{E}+\mathbf{B}$ & $\mathbf{E}+\mathbf{U}$ & $\mathrm{E}+\mathrm{U}+\mathrm{B5}$ & $\mathrm{E}+\mathrm{U}+\mathrm{B} 10$ & $\mathrm{E}+\mathrm{U}+\mathrm{B} 15$ \\
\hline $\mathbf{E}$ & - & $<0.001$ & $<0.001$ & $<0.05$ & $<0.001$ & $<0.001$ \\
\hline $\mathbf{E}+\mathbf{B}$ & & - & $>0.05$ & $<0.01$ & $<0.001$ & $<0.001$ \\
\hline $\mathbf{E}+\mathbf{U}$ & & & - & $>0.05$ & $<0.001$ & $>0.05$ \\
\hline $\mathrm{E}+\mathrm{U}+\mathrm{B5}$ & & & & - & $<0.001$ & $<0.05$ \\
\hline $\mathbf{E}+\mathbf{U}+\mathbf{B} 10$ & & & & & - & $<0.001$ \\
\hline E+U+B15 & & & & & & - \\
\hline
\end{tabular}

$\mathrm{E}=$ effluent de manioc, $\mathrm{E}+\mathrm{B}=$ effluents de manioc $+10 \mathrm{~kg}$ de bouse de vache, $\mathrm{E}+\mathrm{U}=$ effluent de manioc + urine, $\mathrm{E}+\mathrm{U}+\mathrm{B} 5$ $=$ effluents de manioc + urine $+5 \mathrm{~kg}$ de bouse de vache, $\mathrm{E}+\mathrm{U}+\mathrm{B} 10=$ effluents de manioc + urine $+10 \mathrm{~kg}$ de bouse de vache, $\mathrm{E}+\mathrm{U}+\mathrm{B} 15=$ effluents de manioc + urine $+15 \mathrm{~kg}$ de bouse de vache.

\section{DISCUSSION}

Les résultats d'analyse montrent que l'ensemble des digesteurs ont fonctionné dans la gamme de la fermentation mésophile (24 et $35^{\circ} \mathrm{C}$ ). Les essais ont eu lieu entre juillet et octobre, et à cette période de l'année, la température ambiante avoisine les $30{ }^{\circ} \mathrm{C}$. Selon La Farge (1995), les systèmes mésophiles sont les plus courants et les mieux maîtrisés. Ces systèmes résistent mieux aux variations de température et conviennent suffisamment aux systèmes de petite et moyenne tailles utilisés dans le secteur agroalimentaire (DeBruyn et Hilborn, 2007).
Relativement aux $\mathrm{pH}$, les résultats montrent que pour les réacteurs sans urine (1 et 2), les valeurs initiaux sont de 3 pour le digesteur à effluents de manioc (1) et 4 pour le digesteur à effluents de manioc plus bouse de vache (2). En fin d'expérimentation, les valeurs du $\mathrm{pH}$ dans ces deux digesteurs sont restées acides alors que celles des digesteurs avec urine sont passées d'acides à basiques. Chuzel et al. (1995) et Colin et al. (2007) relèvent que les effluents de manioc sont acides en raison de leur composition (richesse en amidon, présence de composés cyanogéniques). De plus, l'acidité des 
effluents bruts observés avant l'alimentation des digesteurs serait en partie due à l'étape de fermentation réalisée au cours du processus de fabrication de l'attiéké. En effet, selon Raimbault (1995), Djoulde (2003) et Oguntoyinbo (2007), la baisse du pH, dans le cas de la production des produits fermentés, s'expliquerait par l'activité de la microflore lactique. Ces microorganismes agissent sur l'amidon qu'ils hydrolysent pour produire des acides lactique et acétique. Djoulde (2003) indique par ailleurs que l'activité amylolytique observée au cours de la fermentation permet de transformer directement l'amidon de manioc en acide lactique. En outre, l'acidité persistante observée au cours du processus de dégradation anaérobie dans les digesteurs sans urine pourrait aussi s'expliquer par l'accumulation des acides gras volatiles, de l'hydrogène mis en place pendant la phase hydrolytique et acidogène.

L'apport d'urine humaine, qui a un caractère basique (Vinneräs, 2002; Kpata, 2005), à l'effluent de manioc acide (Mahan, 2004 ; Kpata, 2005 ; Ubalua, 2007) dans les digesteurs 3, 4, 5, et 6 a rehaussé le $\mathrm{pH}$ dans ces derniers milieux. Les variations de $\mathrm{pH}$ dans ces digesteurs ajustés à l'urine humaine indiquent le passage de la phase acidogène à la phase méthanogène comme observé par Ahring et al. (1995) et Parawira et al. (2006). Selon Moletta (2005), Parawira et al. (2006) et Colin et al. (2007), le pH optimal pour la biodigestion anaérobie se situe entre 6,4 et 7,2 dans le processus de biométhanisation. La légère chute des valeurs du $\mathrm{pH}$ observée dans les premiers jours de l'expérimentation (de 7 à 6,75 pour le réacteur 3 , de 7 à 6,72 pour le réacteur 4 , de 7,01 à 6,61 pour le réacteur 5 et de 7,03 à 6,46 pour le réacteur 6) pourrait s'expliquer par la formation des acides organiques et des acides gras volatils lors de la dégradation de la matière organique
(Kalloum et al., 2007). Cette chute n'a cependant pas eu d'effet inhibiteur sur la méthanisation dans ces digesteurs. La stabilisation du $\mathrm{pH}$ dans la gamme optimale serait due à la présence des bactéries acétogènes qui dissocient les acides organiques (acide lactique, acide acétique, acides propionique...) produits tels que souligné par La Farge (1995) et Colin et al. (2007).

La production de biogaz a été observée dans tous les digesteurs. Les volumes de gaz produits varient de 0,4 à $9 \mathrm{dm}^{3}$ pour le digesteur 1 , de 6,5 à $43,9 \mathrm{dm}^{3}$ pour le réacteur 2 , de 1,6 à $60 \mathrm{dm}^{3}$ pour le réacteur 3 , de 4,4 à $21,12 \mathrm{dm}^{3}$ pour le digesteur 4 , de 8,7 à 81,8 $\mathrm{dm}^{3}$ pour le réacteur 5 et de 1,74 à $52,24 \mathrm{dm}^{3}$ pour le digesteur 6 . Les quantités de biogaz produites sont significativement plus faibles $(\mathrm{p}<0.05)$ dans les réacteurs sans urine que dans les digesteurs avec urine. Au niveau de la qualité du gaz, les six digesteurs présentent des différences très remarquables au niveau du test d'inflammabilité. En effet, le test d'inflammabilité est positif pour les digesteurs tamponnés avec l'urine humaine. Par contre, dans les digesteurs sans urine, ce test est négatif sur toute la période de leur fonctionnement. La production élevée et l'inflammabilité du biogaz dans les digesteurs neutralisés à l'urine humaine au démarrage de l'expérimentation serait dû au pH quasi-neutre du milieu réactionnaire. $\mathrm{Ce} \mathrm{pH}$ neutre aurait permis d'amorcer la méthanogénèse dans les réacteurs. Selon Prescot et al. (2002), Guiraud (2003), Kalloum et al. (2006) et Kalloum et al. (2007), le pH neutre est favorable au développement des bactéries méthanogènes responsables de la formation du biogaz.

\section{Conclusion}

Cette étude fait ressortir que l'ajustement du $\mathrm{pH}$ avant le démarrage du traitement des effluents de manioc issus de la 
filière de fabrication de l'attiéké par digestion anaérobie, est capital pour l'optimisation du processus de biométhanisation. Les résultats de l'étude montrent que l'urine humaine pourrait être utilisée en remplacement des produits chimiques généralement utilisés pour l'ajustement du pH au cours de la biodigestion, notamment celle des effluents de manioc qui présentent une acidité très élevée. De plus, il est recommandé d'ensemencer le milieu réactionnel avec la bouse de vache pour booster la production de biogaz.

\section{REFERENCES}

Ahring BK, Sandberg M, Angelidaki I. 1995. Des acides gras volatils comme indicateurs de déséquilibre processus dans des digesteurs anaérobies. Applied Microbiology and Biotechnology, 43: 559-565.

Amir S. 2005. Contribution à la valorisation de boues de stations d'épuration par compostage : devenir des micropolluants métalliques et organiques et bilan humique du compost. Thèse de Doctorat, Ecole Nationale Supérieure Agronomie, Institut National Polytechnique de Toulouse, France, p. 341.

Asiedu JJ. 1991. La Transformation des Produits Agricoles en Zone Tropicale. CTA : Karthala.

Bouallagui H, Lahdheb H, Ben Romdan E, Rachdi B, Hamdi M. 2009. Improvement of fruit and vegetable waste anaerobic digestion performance and stability with co-substrates addition. Journal of Environmental Management, 90: 18441849.

Chuzel G, Perez D, Dufour D, Alarcon F. 1995. Amélioration d'un système d'extraction par voie humide d'amidon de manioc. In Transformation Alimentaire $d u$ Manioc, Agbor ET, Brauman A,
Griffon D, Trèche S (eds). Editions ORSTOM: Paris; 637-647.

Colin X, Farinet JL, Rojas O, Alazard D. 2007. Anaerobic treatment of cassava starch extraction wastewater using a horizontal flow filter with bamboo as support. Bioresource Technology, 98: 1602-1607.

Cresson R. 2006. Etude du démarrage de procédés intensifs de méthanisation Impact des conditions hydrodynamiques et de la stratégie de montée en charge sur la formation et l'activité du biofilm. Thèse de Doctorat, Université Montpellier II, France, p. 272.

Davidsson A, Lövstedt C, La Cour Jansen J, Gruvberger C, Aspegren H. 2008. Codigestion of grease trap sludge and sewage sludge. Waste Management, 28: 986-992.

DeBruyn J, Hilborn D. 2007. Rudiments de la digestion anaérobie. Fiche technique $\mathrm{N}^{\circ}$ 07-058 AGDEX 720/400.

Djoulde DR. 2003. Mise au point d'un ferment mixte destiné à la bioconversion des tubercules de manioc cyanogène. Thèse de doctorat, Université de Ngaoundéré, Cameroun, p. 200.

Fountoulakis MS, Petousi I, Manios T. 2010. Co-digestion of sewage sludge with glycerol to boost biogas production. Waste Management, 30: 1849-1853.

Gomez X, Moran A, Cuetos MJ, Sanchez ME. 2006. The production of hydrogen by dark fermentation of municipal solid wastes and slaughterhouse waste: a twophase process. Journal of Power Sources, 157: 727-732.

Guiraud JP. 2003. Microbiologie Alimentaire. Edition Dunod: Paris.

Hammer O, Harper DAT, Ryan PD. 2001. Paleontological Statistics Software Package for Education and Data Analysis. Paleontologica Electronica, 4(1): 1-9. 
Héteu TP, Martin J. 2003. Conversion biochimique de la biomasse: Aspects Technologiques et Environnementaux. Unité TERM, Université catholique de Louvain (Belgique), Working paper $\mathrm{N}^{\circ} 3$.

Ihedioha JI. 2002. The clinicopathologic significance of enriching grated cassava mash with red palm oil in the production of gari. Plant Foods for Human Nutrition, 57(3-4): 295-305.

Kakou C. 2000. Optimisation des conditions d'application d'une méthode de conservation longue durée de la pâte de manioc (Manihot esculenta, Crantz) en vue d'améliorer la qualité alimentaire de l'attiéké et du placali. Thèse de Doctorat troisième cycle, Université de Cocody, Côte d'Ivoire, p. 123.

Kalloum S, Boubdessalem H, Touzi A, Iddou A. 2006. La Digestion Anaérobie des boues des stations d'épuration: une opportunité énergétique et environnementale. Mémoire de Magister, Ecole Nationale Supérieure des Enseignements Techniques, Oran, Algérie.

Kalloum S, Khelafi M, Djaafri M, Tahri A, Touzi A. 2007. Etude de l'influence du $\mathrm{pH}$ sur la production du biogaz à partir des déchets ménagers. Revue des Energies Renouvelables, 10(4): 539-543.

Kalloum S, Bouabdessalem H, Touzi A, Iddou A, Ouali MS. 2011. Biogas production from the sludge of the municipal wastewater treatment plant of Adrar city (southwest of Algeria). Biomass and Bioenergy, 35: 2554-2560.

Kpata NE. 2005. Comparaison de la biodigestion anaérobie des effluents issus de la fabrication d'attiéke fertilisé et non fertilisé à l'urine humaine. Mémoire de DEA, Université d'Abobo-Adjamé, Côte d'Ivoire, p. 40.
La Farge De B. 1995. Le Biogaz, Procédé de Fermentation Méthanique. Edition Masson: Paris.

Le Hyaric R, Canler JP, Barillon B, Naquin P, Gourdon R. 2010. Pilot-scale anaerobic digestion of screenings from wastewater treatment plants. Bioresource Technology, 101: 9006-9011.

Luostarinen S, Luste S, Sillanpää M. 2009. Increased biogas production at wastewater treatment plants through codigestion of sewage sludge with grease trap sludge from a meat processing plant. Bioresource Technology, 100: 79-85.

Mahan V. 2004. Etude de l'épuration des effluents issus des unités de production d'attiéké. Mémoire de DEA, Université d'Abobo-Adjamé, Côte d'Ivoire, p. 38.

Mata-Alvarez J, Macé S, Llabrés P. 2000. Anaerobic digestion of organic solid wastes. An overview of research achievements and perspectives. Bioresource Technology, 74: 3-16.

Moletta M. 2005. Caractérisation de la Diversité Microbienne Aéroportée des Biogaz. Thèse de Doctorat, Université de Montpellier II, France, p. 195.

Montusiewicz A, Lebiocka M. 2011. Codigestion of intermediate landfill leachate and sewage sludge as a method of leachate utilization. Bioresource Technology, 102: 2563-2571.

Oguntoyinbo FA. 2007. Identification and functional properties of dominant lactic acid bacteria isolated at different stages of solid state fermentation of cassava during traditional gari production. World Journal of Microbiology and Biotechnology, 23: 1425-1432.

Parawira W, Murto M, Zvauya R, Mattiasson B. 2006. Comparative Performance of a UASB Reactor and an Anaerobic PackedBed Reactor when treating potato waste 
leachate. Renewable Energy, 31(6): 893903.

Poh PE, Chong MF. 2009. Development of anaerobic digestion methods for palm oil mill effluent (POME) treatment. Bioresource Technology, 1-9.

Prescott LM, Harley JP, Klein DA. 2002. Microbiology ( $5^{\text {th }}$ edn). The McGraw-Hill Companies, Inc: North America.

Raimbault M. 1995. Importance des bactéries lactiques dans les fermentations du manioc. In Transformation Alimentaire du Manioc, Agbor ET, Brauman A, Griffon D, Trèche $S$ (eds). Editions ORSTOM: Paris; 259-275.
Ubalua AO. 2007. Cassava wastes: treatment options and value addition alternatives. African Journal of Biotechnology, 18(6): 2065-2073.

Vinneräs B, 2002. Possibilities for sustainable nutrient recycling by faecal separation combined with urine diversion. $\mathrm{PhD}$ thesis, Swedish University of Agricultural Sciences, Uppsala, Swedish, p. 258.

Zhu Z, Hsueh KM, He Q. 2011. Enhancing biomethanation of municipal waste sludge with grease trap waste as a cosubstrate. Renewable Energy, 36: 18021807. 